\title{
Impacto de uma intervenção de educação alimentar no perfil nutricional e padrão de consumo alimentar em mulheres no climatério
}

Helena de Matos Gaira*, Francisca Maria Assmann Wichmann ${ }^{* *}$, Fabiana Assmann Poll ${ }^{* * *}$

\section{Resumo}

Estratégias de intervenção, no âmbito da educação alimentar e nutricional, podem contribuir para melhoria do padrão de consumo alimentar e estado nutricional de mulheres no climatério, resultando em benefícios à saúde e qualidade de vida. O objetivo do estudo foi avaliar o impacto de uma intervenção nutricional com 2 meses de duração, baseada em estratégias de educação alimentar e nutricional, no estado nutricional, risco cardiovascular, sintomas do climatério, consumo alimentar e no estilo de vida de mulheres adultas. Trata-se de um estudo quase experimental. Coletaram-se dados, pré e pós intervenção, relacionados ao estado nutricional (peso, altura, índice de massa corporal (IMC) e circunferência da cintura (CC)), estilo de vida, satisfação e padrão de peso corporal e frequência de consumo alimentar. Os sintomas do climatério foram avaliados através do Menopause Rating Scale (MRS). Os dados foram analisados no Programa Statistical Package for Social Science (SPSS). Totalizaram-se 16 mulheres, com idade média de 54 55,24 anos, sendo que $62,5 \%$ referiram aumento do peso nos úl- timos anos, e 75\% manifestaram insatisfação corporal. Houve redução significativa da CC $(p=0,006)$ e da classificação do IMC $(p=0,046)$, do pré para o pós intervenção. Verificou-se aumento na frequência de ingestão de produtos lácteos, ovos, feijão, grãos, e redução do pão branco. Quanto ao estilo de vida e sintomas de climatério, não foram observadas mudanças significativas. A intervenção nutricional com 2 meses de duração mostrou impacto positivo em hábitos de consumo alimentar e estado nutricional de mulheres em fase de climatério.

Palavras-chave: Climatério; Educação alimentar e nutricional; Estado nutricional.

\section{Introdução}

No processo de viver as mulheres buscam sentir-se bem consigo e no meio em que vivem a fim de compreender-se, aceitar suas limitações e medos, conhecer suas potencialidades para uma melhor qualidade de vida (ZAMPIERI et

* Graduanda em Nutrição (UNISC). Curso de Nutrição- UNISC.

** Doutora em Desenvolvimento Regional (UNISC). Departamento de Educação Física e Saúde - Curso de Nutrição - Universidade de Santa Cruz do Sul (UNISC).

*** Doutora em Saúde da Criança e do Adolescente (UFRGS). Departamento de Educação Física e Saúde- Curso de Nutrição - Universidade de Santa Cruz do Sul (UNISC).

http://dx.doi.org/10.5335/rbceh.v17i1.9635 
$a l ., 2009)$. O climatério é um momento de transição do período reprodutivo para o não reprodutivo, que acontece na faixa entre os 40 e 65 anos da mulher (NOSSE; MOREIRA; ANDRADE, 2009).

A Organização Mundial da Saúde (OMS) (1996) define climatério como um período de envelhecimento, que determina o fim dos ciclos menstruais. $\mathrm{E}$ a menopausa é reconhecida apenas um ano depois do último ciclo menstrual. Nele, ocorrem modificações clínicas e metabólicas no corpo da mulher, pela perda da função folicular ovariana e redução do estrógeno. Isso ocorre gradativamente, causando sinais e sintomas vaso motores, os fogachos. As alterações emocionais causadas pelo hipoestrogenismo interferem na síntese de neurotransmissores, modificando o comportamento, labilidade emocional e dificuldade de memorizar. Durante esse período, outra mudança que ocorre é o aumento ponderal de peso (aproximadamente 0,8 kg por ano) (FERNANDES; BARACAT; LIMA, 2004), sendo que depois da menopausa este, pode elevar-se cerca de $20 \%$. A principal causa destes eventos é atribuída ao descontrole alimentar e a dieta inadequada (PEDRO et al., 2003).

No entanto, outros estudos demonstraram que mulheres após a menopausa apresentam um aumento da circunferência abdominal, assim como elevação da gordura corporal (ABDULNOUR et al., 2012). Cerca de $60 \%$ das mulheres na fase do climatério apresentam um rápido ganho de peso. Fato que pode ter relação com o estilo de vida em sociedades indus- trializadas, que geralmente promove um acréscimo de peso corporal com o avanço da idade, além de estar associado à redução da taxa de metabolismo basal e da prática de exercícios físicos (NELSI; CORRADINI; FELICIO, 2008).

A obesidade perimenopausal apresenta forte relação com morbidades, com destaque às doenças cardiovasculares. Portanto, as mulheres neste período precisam adquirir hábitos alimentares saudáveis, que contribuam para perda $\mathrm{e}$ manutenção de peso corporal (MONTEIRO; RIETHER; BURINI, 2004). Existe evidência de que nessa fase da vida $50 \%$ da população feminina apresenta síndrome metabólica (NETO et al., 2010), e que no período inicial do climatério há um aumento progressivo de peso, o que eleva o risco de morbidades. Além disso, mulheres obesas pós-menopausa tem 50\% maior risco de câncer do que mulheres não obesas (GU et al., 2011).

Nesse sentido estratégias de intervenção nutricional podem melhorar o padrão do consumo alimentar e as medidas antropométricas resultando em benefícios a saúde física e mental no climatério (GALLON; WENDER, 2012). Para tanto, o objetivo da pesquisa foi avaliar o impacto de uma intervenção nutricional, baseada em estratégias de educação alimentar e nutricional (EAN), com dois meses de duração, sobre o estado nutricional, risco cardiovascular, sintomas do climatério, consumo alimentar e estilo de vida de mulheres adultas. 


\section{Métodos}

Trata-se de um estudo do tipo quase-experimento. A amostragem se deu por conveniência, a partir de divulgação em mídias sociais e na Universidade de Santa Cruz do Sul (UNISC), local de desenvolvimento da pesquisa. A amostra atendeu aos critérios de inclusão, tais como, mulheres com idade entre 40 e 65 anos, que concordaram em participar da pesquisa mediante a assinatura do Termo de Consentimento Livre e Esclarecido e que tinham disponibilidade e condições de deslocamento para participar dos encontros na Universidade no período estabelecido.

A intervenção nutricional teve duração de dois meses, sendo que dados referentes à alimentação, estilo de vida, percepção corporal e estado nutricional e sintomas de climatério foram questionados antes e após esse período.

Os encontros foram conduzidos pelas pesquisadoras responsáveis, em uma noite por semana, e tiveram duração aproximada de 90 minutos, ao longo dos meses de abril e maio de 2019 , junto ao Serviço Integrado de Saúde (SIS) da UNISC.

A intervenção nutricional envolveu técnicas educativas e motivacionais, baseadas em estratégias de EAN. Os temas para os encontros estavam relacionados ao estímulo a escolhas alimentares adequadas para esse momento da vida, visando maior qualidade nutricional e perda de peso, usando como material de apoio o Guia Alimentar para População Brasileira (MINISTÉRIO DA SAÚDE,
2014), seguido da abordagem de uma alimentação consciente, com estratégias do Centro Brasileiro de Mindfull Eating (VIDAL et al, 2012) e de alimentos que contribuem na redução de processos inflamatórios e na saúde intestinal (BERNAUD; RODRIGUES, 2013; IRMÃO; COSTA, 2018).

Esses temas foram desenvolvidos através de rodas de conversas, palestras, com o estabelecimento de metas de mudança para a semana. Cada participante escolhia sua própria meta, conforme sua necessidade naquele momento. Para auxiliar na mudança do comportamento alimentar, no inicio de cada encontro as metas eram discutidas e avaliado o que poderia ser melhorado, ao final de cada encontro, um alimento ou preparação era oferecido para degustação, bem como a receita, indicando os nutrientes e os benefícios para saúde.

Os dados foram coletados a partir da ficha de anamnese que o Curso de Nutrição da UNISC utiliza para os atendimentos e práticas de ensino, pesquisa e extensão no Serviço Integrado de Saúde (SIS). Referiam-se ao estilo de vida (prática de atividade física e hábitos de sono), a aspectos clínicos (uso de medicamentos) e nutricionais (consumo alimentar no último mês); questionou-se a satisfação com peso corporal; e se o peso atual sofreu alguma alteração recente no sentido de aumentar, reduzir ou manter.

Para a avaliação antropométrica foram coletados o peso corporal em quilogramas $(\mathrm{kg})$ e estatura em metros (m). Para verificar o peso foi utilizada a balança eletrônica da marca Welmy ${ }^{\circledR}$, 
com capacidade para $200 \mathrm{~kg}$ e a altura utiliza-se o estadiômetro que a própria balança apresenta. A Circunferência da Cintura (CC) foi aferida no ponto médio entre o último rebordo costal e a crista ilíaca, com fita métrica inelástica da marca Macrofile $\square$. Todas as medidas antropométricas foram realizadas antes e após a intervenção em sala de atendimento individual, com o mínimo de vestuário possível.

Para avaliação do estado nutricional utilizou-se o Índice de Massa Corporal (IMC) classificado pelos critérios da Organização Mundial de Saúde (OMS) (WHO, 1995) e para classificar o risco cardiovascular pela CC adotou-se os parâmetros da OMS (WHO, 1998).

Para avaliação dos sintomas relacionados ao climatério e qualidade de vida, foi utilizado o Menopause Rating Scale (MRS), que é um instrumento validado no Brasil, composto por 11 questões que relatam sintomas divididos em domínios somatovegetativos, psicológicos e urogenitais. Para cada indivíduo o escore total de cada sub-escala resulta da soma da pontuação de cada item da mesma, estando a maior pontuação obtida associada a uma severa sintomatologia e a uma pior qualidade de vida da mulher. Os resultados de pontuação são classificados da seguinte forma: assintomáticos ou escassos - até 4 pontos; leves - de 5 a 8 pontos; moderados - de 9 a 15 pontos; e severos - mais de 16 pontos.
Os dados foram analisados no Programa Statistical Package for Social Science (SPSS). Realizou-se uma estatística descritiva e utilizou-se o teste de McNemar em tabelas 2x2 ou o teste de homogeneidade marginal em tabelas kxk $(\mathrm{k}>2)$ para avaliar se houve mudança na categoria das respostas das pacientes. $\mathrm{O}$ nível de significância foi de $\mathrm{p}<0,05$.

O estudo foi aprovado pelo Comitê de Ética da Universidade de Santa Cruz do Sul sob CAAE nº 03737818.0.0000.5343 e parecer $n^{\circ} 3.054 .977$, e seguiu todos os preceitos éticos preconizados na Resolução 466/12 do Conselho Nacional de Saúde. Todos as participantes assinaram o termo de consentimento livre e esclarecido antes do inicio da pesquisa.

\section{Resultados}

A amostra foi constituída de 16 mulheres, com idade média de $54 \pm 5,24$ anos. Não houve perdas amostrais ao longo da intervenção. A caracterização da amostra quanto ao estado nutricional, risco cardiovascular, satisfação corporal e classificação do escore do MRS, antes e após a intervenção, está apresentada na Tabela 1. 
Tabela 1: Caracterização da amostra segundo o estado nutricional, risco cardiovascular, satisfação corporal e escore do MRS antes e após a intervenção nutricional (n=16).

\begin{tabular}{|c|c|c|c|c|c|}
\hline \multirow{2}{*}{ Variável } & \multicolumn{2}{|c|}{ Antes da intervenção } & \multicolumn{2}{|c|}{ Depois da intervenção } & \multirow{2}{*}{$p^{*}$} \\
\hline & $\mathrm{N}$ & $\%$ & $\mathrm{~N}$ & $\%$ & \\
\hline Peso (kg) & \multicolumn{2}{|c|}{$78,16 \pm 13,74$} & \multicolumn{2}{|c|}{$76,72 \pm 13,76$} & $<0,001$ \\
\hline $\mathrm{IMC}\left(\mathrm{kg} / \mathrm{m}^{2}\right)$ & \multicolumn{2}{|c|}{$28,98 \pm 5,28$} & \multicolumn{2}{|c|}{$28,45 \pm 5,30$} & $<0,001$ \\
\hline \multicolumn{5}{|l|}{ Classificação do IMC } & 0,046 \\
\hline Desnutrição & & 0,0 & 0 & 0,0 & \\
\hline Eutrófico & & 31,3 & 6 & 37,5 & \\
\hline Sobrepeso & & 18,8 & 5 & 31,3 & \\
\hline Obesidade & & 50,0 & 5 & 31,3 & \\
\hline Circunferência da cintura $(\mathrm{cm})$ & \multicolumn{2}{|c|}{$93,81 \pm 12,05$} & \multicolumn{2}{|c|}{$91,62 \pm 10,81$} & 0,006 \\
\hline \multicolumn{5}{|c|}{ Classificação da Circunferência da cintura } & 1,000 \\
\hline Sem risco & & 12,5 & 3 & 18,8 & \\
\hline Com risco & & 87,5 & 13 & 82,3 & \\
\hline \multicolumn{5}{|l|}{ Satisfação corporal } & 1,000 \\
\hline Não & & 75 & 12 & 75 & \\
\hline Sim & & 25 & 4 & 25 & \\
\hline \multicolumn{5}{|l|}{ Sintomas de climatério } & 0,527 \\
\hline Assintomático & & 6,3 & 2 & 12,5 & \\
\hline Leve & & 28,8 & 2 & 12,5 & \\
\hline Moderado & & 37,5 & 7 & 43,8 & \\
\hline Severo & & 37,5 & 5 & 31,3 & \\
\hline
\end{tabular}

*teste t de Student pareado para variáveis contínuas e teste de McNemar ou o teste de homogeneidade marginal para variáveis categóricas. IMC: índice de massa corporal. MRS: Menopause Rating Scale.

A auto percepção referente ao peso corporal foi questionada antes da intervenção, e observou-se que $37,5 \%$ das mulheres referiram que seu peso era estável e $62,5 \%$, que o peso estava aumentando. A frequência de consumo alimentar das mulheres nos dois momentos avaliados está disposta na Tabela 2. 
Tabela 2: Frequência de consumo alimentar das mulheres antes e após a intervenção nutricional

(continua...)

\begin{tabular}{|c|c|c|c|c|c|}
\hline \multirow{2}{*}{ Variável } & \multicolumn{2}{|c|}{ Antes da intervenção } & \multicolumn{2}{|c|}{ Depois da intervenção } & \multirow{2}{*}{$\mathrm{p}^{*}$} \\
\hline & $\mathrm{N}$ & $\%$ & $\mathrm{~N}$ & $\%$ & \\
\hline Hortaliças coloridas & & & & & 0,414 \\
\hline Nunca & 0 & 0,0 & 0 & 0,0 & \\
\hline 1-2 vezes por semana & 2 & 12,5 & 1 & 6,3 & \\
\hline 3-4 vezes por semana & 4 & 25,0 & 4 & 25,0 & \\
\hline Diário & 10 & 62,5 & 11 & 68,8 & \\
\hline Frutas & & & & & 0,317 \\
\hline Nunca & 0 & 0,0 & 0 & 0,0 & \\
\hline 1-2 vezes por semana & 1 & 6,3 & 2 & 12,5 & \\
\hline 3-4 vezes por semana & 3 & 18,8 & 2 & 12,5 & \\
\hline Diário & 12 & 75,0 & 12 & 75,0 & \\
\hline Lácteos & & & & & 0,021 \\
\hline Nunca & 2 & 12,5 & 1 & 6,3 & \\
\hline $1-2$ vezes por semana & 5 & 31,3 & 2 & 12,5 & \\
\hline 3-4 vezes por semana & 3 & 18,8 & 4 & 25,0 & \\
\hline Diário & 6 & 37,5 & 9 & 56,3 & \\
\hline Carnes & & & & & 0,366 \\
\hline Nunca & 1 & 6,3 & 2 & 12,5 & \\
\hline 1-2 vezes por semana & 0 & 0,0 & 0 & 0,0 & \\
\hline 3-4 vezes por semana & 2 & 12,5 & 2 & 12,5 & \\
\hline Diário & 13 & 81,3 & 12 & 75,0 & \\
\hline Peixes & & & & & 0,257 \\
\hline Nunca & 0 & 0,0 & 0 & 0,0 & \\
\hline 1-2 vezes por semana & 0 & 0,0 & 2 & 12,5 & \\
\hline 3-4 vezes por semana & 10 & 62,5 & 9 & 56,3 & \\
\hline Diário & 6 & 37,5 & 5 & 31,3 & \\
\hline Ovos & & & & & 0,007 \\
\hline Nunca & 0 & 0,0 & 0 & 0,0 & \\
\hline 1-2 vezes por semana & 5 & 31,3 & 1 & 6,3 & \\
\hline 3-4 vezes por semana & 8 & 50,0 & 7 & 43,8 & \\
\hline Diário & 0 & 0,0 & 8 & 50,0 & \\
\hline Embutidos & & & & & 0,206 \\
\hline Nunca & 6 & 37,5 & 8 & 50,0 & \\
\hline 1-2 vezes por semana & 7 & 43,8 & 7 & 43,8 & \\
\hline 3-4 vezes por semana & 3 & 18,8 & 1 & 6,3 & \\
\hline Diário & 0 & 0,0 & 0 & 0,0 & \\
\hline
\end{tabular}


Impacto de uma intervenção de educação alimentar no perfil nutricional e padrão de consumo alimentar ...

(continuação...)

Feijão

Nunca

1-2 vezes por semana

3-4 vezes por semana

Diário

Frituras

Nunca

1-2 vezes por semana

3-4 vezes por semana

Diário

Doces em geral

Nunca

1-2 vezes por semana

3-4 vezes por semana

Diário

Açúcar

Nunca

1-2 vezes por semana

3-4 vezes por semana

Diário

Adoçante

Nunca

1-2 vezes por semana

3-4 vezes por semana

Diário

\section{Café}

Nunca

1-2 vezes por semana

3-4 vezes por semana

Diário

Chimarrão

Nunca

1-2 vezes por semana

3-4 vezes por semana

Diário

Suco artificial

Nunca

1-2 vezes por semana

3-4 vezes por semana

Diário

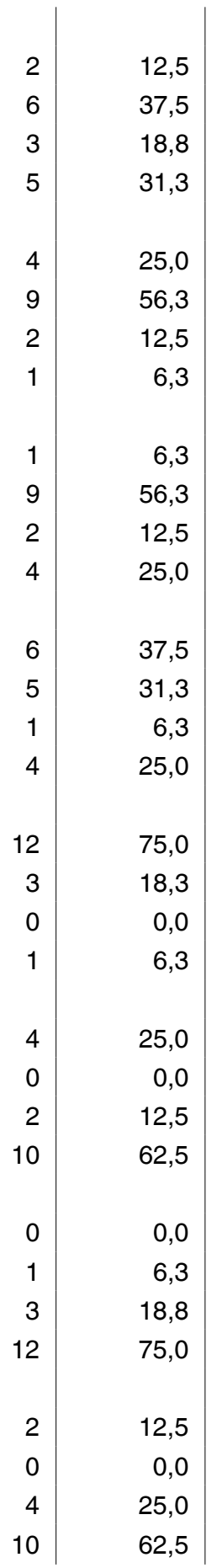

0,014

6,3

25,0

25,0

43,8

31,3

56,3

6,3

6,3

0,059

25,0

50,0

18,8

6,3

0,371

43,8

31,3

12,5

12,5

0,414

81,3

12,5

6,3

0,0

0,567

12,5

18,8

25,0

43,8

1,000

0,0

12,5

6,3

81,3

0,109 
(conclusão...)

Refrigerantes
Nunca
1-2 vezes por semana
3-4 vezes por semana
Diário

Bebidas alcoólicas

Nunca

1-2 vezes por semana

3-4 vezes por semana

Diário

Biscoitos

Nunca

1-2 vezes por semana

3-4 vezes por semana

Diário

Cereais integrais

Nunca

1-2 vezes por semana

3-4 vezes por semana

Diário

Grãos (chia, gergelim, linhaça, amaranto, quinoa)

Nunca

1-2 vezes por semana

13-4 vezes por semana

Diário

Pão branco

Nunca

1-2 vezes por semana

3-4 vezes por semana

Diário

Pão integral

Nunca

1-2 vezes por semana

3-4 vezes por semana

Diário

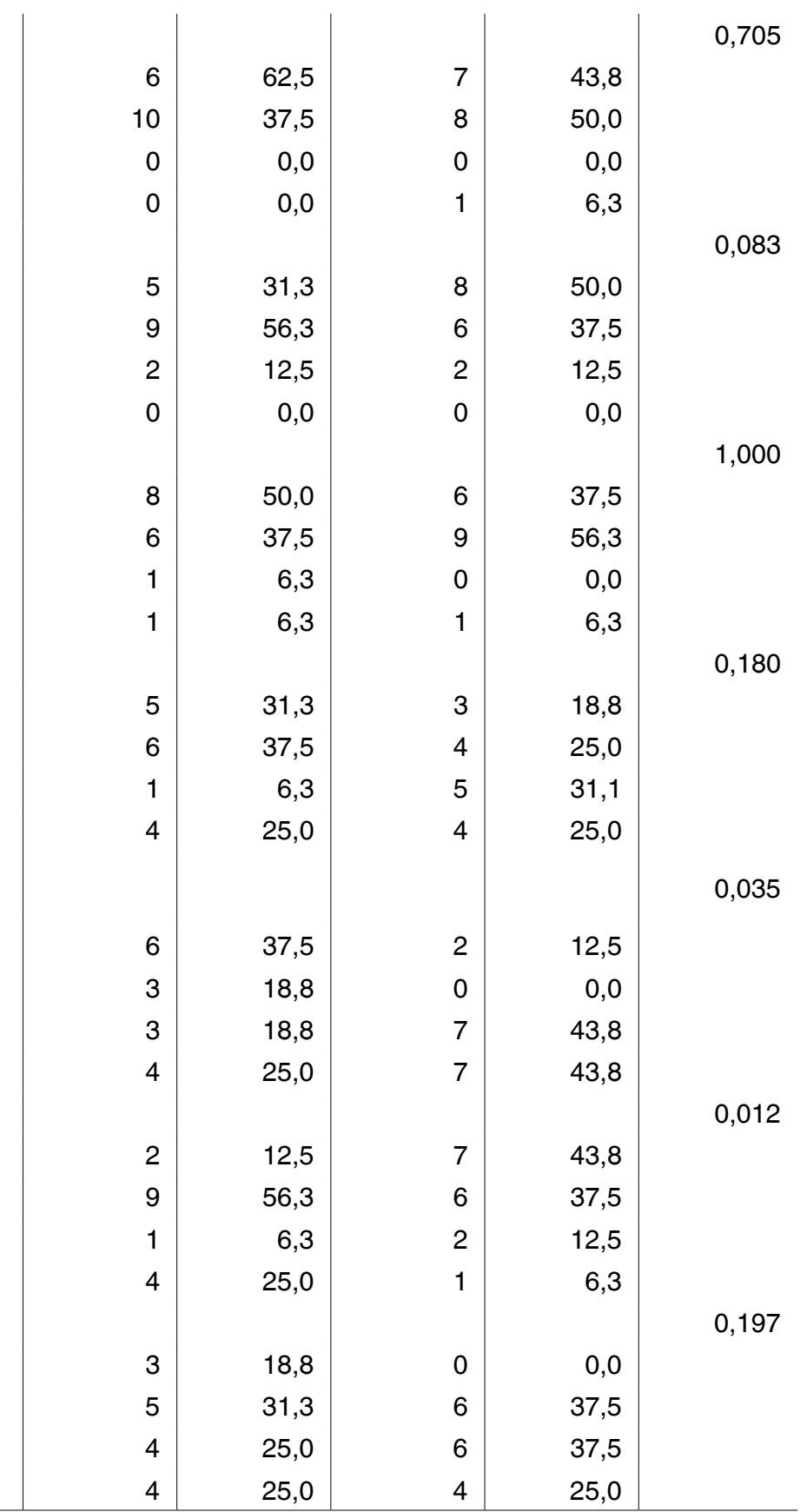

$\mathrm{N}$ (número de participantes)*teste de homogeneidade marginal 
Não houve modificações significativas na comparação entre os resultados antes e após a intervenção nutricional quanto as variáveis de estilo de vida (Tabela 3).

Tabela 3: Associação de fatores relacionados ao estilo de vida, antes e após a intervenção nutricional.

\begin{tabular}{|c|c|c|c|c|c|}
\hline \multirow{2}{*}{ Variável } & \multicolumn{2}{|c|}{ Antes da intervenção } & \multicolumn{2}{|c|}{ Depois da intervenção } & \multirow{2}{*}{$\mathrm{p}^{\star}$} \\
\hline & $\mathrm{N}$ & $\%$ & $\mathrm{~N}$ & $\%$ & \\
\hline Horas de sono & & & & & 1,000 \\
\hline Menos de $6 \mathrm{~h}$ & 2 & 12,5 & 2 & 12,5 & \\
\hline Mais de $6 \mathrm{~h}$ & 14 & 87,5 & 14 & 87,5 & \\
\hline Qualidade do sono & & & & & 1,000 \\
\hline Bom & 11 & 68,8 & 12 & 75,0 & \\
\hline Ruim & 5 & 31,3 & 4 & 25,0 & \\
\hline Medicamentos & & & & & 1,000 \\
\hline Não & 6 & 37,5 & 6 & 37,5 & \\
\hline Sim & 10 & 62,5 & 10 & 62,5 & \\
\hline Atividade física & & & & & 0,500 \\
\hline Não & 7 & 43,8 & 5 & 31,3 & \\
\hline Sim & 9 & 56,3 & 11 & 68,8 & \\
\hline
\end{tabular}

*teste de McNemar.

Houve uma redução no consumo do pão branco $(\mathrm{p}=0,012)$ e aumento no consumo de produtos lácteos $(\mathrm{p}=0,021)$, ovos $(\mathrm{p}=0,007)$, feijão $(\mathrm{p}=0,014)$, grãos e sementes $(\mathrm{p}=0,035)$.

Os sintomas do climatério que constam no MRS, antes e após a intervenção, estão dispostos na Tabela 4. 
Tabela 4: Sintomas relacionados ao climatério obtidos pelo MRS, antes e após a intervenção nutricional

(continua...)

\begin{tabular}{|c|c|c|c|c|c|}
\hline \multirow{2}{*}{ Variável } & \multicolumn{2}{|c|}{ Antes da intervenção } & \multicolumn{2}{|c|}{ Depois da intervenção } & \multirow[t]{2}{*}{$p^{*}$} \\
\hline & $\mathrm{N}$ & $\%$ & $\mathrm{~N}$ & $\%$ & \\
\hline Falta de ar & & & & & 1,000 \\
\hline Nenhum & 6 & 37,5 & 4 & 25,0 & \\
\hline Pouco severo & 4 & 25,0 & 6 & 37,5 & \\
\hline Moderado & 2 & 12,5 & 4 & 25,0 & \\
\hline Severo & 4 & 25,0 & 2 & 12,5 & \\
\hline Muito severo & 0 & 0,0 & 0 & 0,0 & \\
\hline Mal-estar & & & & & 0,366 \\
\hline Nenhum & 7 & 43,8 & 6 & 37,5 & \\
\hline Pouco severo & 3 & 18,8 & 8 & 50,0 & \\
\hline Moderado & 5 & 31,3 & 1 & 6,3 & \\
\hline Severo & 1 & 6,3 & 1 & 6,3 & \\
\hline Muito severo & 0 & 0,0 & 0 & 0,0 & \\
\hline Problemas de sono & & & & & 1,000 \\
\hline Nenhum & 5 & 31,1 & 6 & 37,5 & \\
\hline Pouco severo & 5 & 31,3 & 2 & 12,5 & \\
\hline Moderado & 2 & 12,5 & 5 & 31,3 & \\
\hline Severo & 3 & 18,8 & 2 & 12,5 & \\
\hline Muito severo & 1 & 6,3 & 1 & 6,3 & \\
\hline Problemas de ânimo, depressivos & & & & & 0,617 \\
\hline Nenhum & 4 & 25,0 & 3 & 18,8 & \\
\hline Pouco severo & 4 & 25,0 & 7 & 43,8 & \\
\hline Moderado & 4 & 25,0 & 4 & 25,0 & \\
\hline Severo & 4 & 25,0 & 1 & 6,3 & \\
\hline Muito severo & 0 & 0,0 & 1 & 6,3 & \\
\hline Irritabilidade & & & & & 0,705 \\
\hline Nenhum & 2 & 12,5 & 3 & 18,8 & \\
\hline Pouco severo & 7 & 43,8 & 7 & 43,8 & \\
\hline Moderado & 4 & 25,0 & 4 & 25,0 & \\
\hline Severo & 3 & 18,8 & 1 & 6,3 & \\
\hline Muito severo & 0 & 0,0 & 1 & 6,3 & \\
\hline
\end{tabular}




\begin{tabular}{|c|c|c|c|c|c|}
\hline Ansiedade & & & & & 0,056 \\
\hline Nenhum & 2 & 12,5 & 6 & 37,5 & \\
\hline Pouco severo & 6 & 37,5 & 6 & 37,5 & \\
\hline Moderado & 4 & 25,0 & 3 & 18,8 & \\
\hline Severo & 3 & 18,8 & 0 & 0,0 & \\
\hline Muito severo & 1 & 6,3 & 1 & 6,3 & \\
\hline Esgotamento físico e mental & & & & & 0,221 \\
\hline Nenhum & 3 & 18,8 & 6 & 37,5 & \\
\hline Pouco severo & 6 & 37,5 & 4 & 25,0 & \\
\hline Moderado & 4 & 25,0 & 5 & 31,3 & \\
\hline Severo & 2 & 12,5 & 0 & 0,0 & \\
\hline Muito severo & 1 & 6,3 & 1 & 6,3 & \\
\hline Falta de desejo sexual & & & & & 1,000 \\
\hline Nenhum & 6 & 37,5 & 6 & 37,5 & \\
\hline Pouco severo & 4 & 25,0 & 4 & 25,0 & \\
\hline Moderado & 3 & 18,8 & 3 & 18,8 & \\
\hline Severo & 1 & 6,3 & 1 & 6,3 & \\
\hline Muito severo & 2 & 12,5 & 2 & 12,5 & \\
\hline Problemas de bexiga & & & & & 0,847 \\
\hline Nenhum & 9 & 56,3 & 9 & 56,3 & \\
\hline Pouco severo & 3 & 18,8 & 3 & 18,8 & \\
\hline Moderado & 1 & 6,3 & 2 & 12,5 & \\
\hline Severo & 2 & 12,5 & 1 & 6,3 & \\
\hline Muito severo & 1 & 6,3 & 1 & 6,3 & \\
\hline Ressecamento vaginal & & & & & 0,763 \\
\hline Nenhum & 7 & 43,8 & 8 & 50,0 & \\
\hline Pouco severo & 5 & 31,3 & 3 & 18,8 & \\
\hline Moderado & 2 & 12,5 & 2 & 12,5 & \\
\hline Severo & 1 & 6,3 & 2 & 12,5 & \\
\hline Muito severo & 1 & 6,3 & 1 & 6,3 & \\
\hline Problemas articulares & & & & & 0,343 \\
\hline Nenhum & 2 & 12,5 & 2 & 12,5 & \\
\hline Pouco severo & 8 & 50,0 & 6 & 37,5 & \\
\hline Moderado & 3 & 18,8 & 2 & 12,5 & \\
\hline Severo & 1 & 6,3 & 3 & 18,8 & \\
\hline Muito severo & 2 & 12,5 & 3 & 18,8 & \\
\hline
\end{tabular}

*teste de homogeneidade marginal. 


\section{Discussão}

Ao início da intervenção, $50 \%(\mathrm{n}=8)$ das mulheres foram classificadas obesas, sendo muito semelhante a prevalência encontrada por Silva et al. (2012) em estudo realizado com 32 mulheres com idade média de $52 \pm 5,4$ anos constatou que $53,1 \%$ estavam com obesidade. Porém após a intervenção de EAN, do presente estudo, $31,3 \%$ permaneceram nesse estado nutricional. Em relação à distribuição de gordura corporal, houve predominância de acúmulo na região abdominal, retratando um perfil de obesidade bastante documentado em mulheres no climatério, o que pode ser devido às alterações hormonais impostas por esta fase (NETO et al., 2010; SILVA et al., 2012). Ao final do acompanhamento das $87,5 \%$ que estavam com risco, uma participante $(17,9 \%)$ obteve redução, com menor possibilidade de desencadear doença cardiovascular (DCV), não tendo sido estatisticamente significativa a diminuição da média de CC final em relação à média inicial. Destacando os benefícios da redução da $\mathrm{CC}$, um estudo de meta-análise realizado por Koning et al. (2007) constatou que essa medida pode ser considerada fator de risco para DCV e o aumento de um centímetro da CC pode aumentar em $2 \%$ o risco para $\mathrm{DCV}$, sendo o inverso verdadeiro. Os autores ainda sugerem que a medida da CC deve ser incluída na rotina para avaliação de risco cardiovascular. Houve redução significativa da CC média de 93,81 $\pm 12,04 \mathrm{~cm}$ para $91,62 \pm 10,8 \mathrm{~cm}$ no pós-intervenção. Esses valores foram menores do que o encontrado num estudo com 30 mulheres adultas, em risco cardiovascular, atendidas no Ambulatório de Nutrição de uma Universidade do Norte do Rio Grande do Sul, cuja CC média era de $98,2 \pm 15,9 \mathrm{~cm}$ (MARTINAZZOI et al., 2013). Uma pesquisa desenvolvida na Universidade da Terceira Idade em Amazonas/AM por 40 semanas, com 30 mulheres no climatério, que foram divididas entre grupo de intervenção nutricional (GD) e grupo de intervenção nutricional com exercício físico (GE), verificou uma tendência de redução da CC, após a intervenção, no GD de $4,8 \mathrm{~cm}$, e mais ainda no GE, com $7,6 \mathrm{~cm}$ (MONTEIRO; RIETHER; BURINI, 2004). Os resultados do GD dessa pesquisa foram superiores aos do presente estudo, porém o processo de intervenção se deu por um tempo de duração maior, o que pode favorecer essa resposta.

Quanto ao consumo alimentar observaram-se modificações após a intervenção nutricional, com aumento do consumo de lácteos $(\mathrm{p}=0,021)$, feijão $(\mathrm{p}=0,014)$, grãos (chia, gergelim, linhaça, amaranto, quinoa) ( $\mathrm{p}=0,035), \operatorname{ovos}(0,007)$, e redução do consumo de pão branco $(\mathrm{p}=0,012)$. Monteiro, Riether e Burini (2004) concluíram que a associação de dieta e EAN se mostrou efetiva na promoção de mudanças no consumo habitual de alimentos. Sabe-se que existe a influência positiva das estratégias de intervenção nutricional para prevenção da obesidade, de doenças crônicas e promoção da saúde, pois podem atuar na mudança de hábitos e na melhora do estado nutricional, na medida em que aumentam o conheci- 
mento de práticas alimentares saudáveis (GOMES et al., 2013).

O padrão alimentar do estudo é semelhante ao estudo de Hoffmann et al. (2015), que aponta um maior consumo de frutas, verduras e grãos integrais, sendo muito similar do que é ideal dentre as recomendações do mais recente guia alimentar da população brasileira. Foram observadas pequenas mudanças no consumo alimentar da população, incluindo um aumento no consumo de lácteos, ovos legumes e verduras e uma diminuição no consumo de carnes vermelhas, doces e enlatados, mas sem significância estatística. Os resultados são similares a outros estudos, inclusive no sul do Brasil, que já identificaram esta tendência, ou seja, as mulheres adquirem hábitos alimentares mais saudáveis com o aumento da idade (HOFFMANN et al., 2015).

Os sintomas de climatério não tiveram mudanças significativas no presente estudo, quando comparados antes e após a intervenção. Antes da intervenção $28,8 \%$ referiram presença de sintomas leves, $37,5 \%$ moderados e $37,5 \%$ severos, diferente de um estudo transversal realizado em Caixas do Sul/RS com 615 mulheres na mesma faixa etária do presente estudo, que frequentavam a clínica ginecológica da universidade, e constataram que $34,1 \%$ (IC95\% 30,3- 37,9) das mulheres apresentaram sintomas leves, 29,6\% (IC95\% 25,8-33,1) moderados e $36,3 \%$ (IC95\% 32,4-40,0) graves (BARAZZETTI et al., 2016). Já outro estudo, que envolveu 1884 mulheres entre 40 e 65 anos de idade, realizou uma análise re- trospectiva de questionários preenchidos no atendimento clínico para menopausa ou consulta de saúde sexual entre maio de 2015 e maio de 2017 em Minessottal EUA, obtiveram prevalência menor de sintomas menopausais graves ou muito graves (19\%) (KLING et al., 2019).

Segundo Organização Mundial de Saúde (OMS, 1996) o estilo de vida é um conjunto de hábitos e costumes diários, com influência positiva ou negativa para a saúde. Os hábitos dos indivíduos implicam na qualidade de vida e são objetos de pesquisa e investigação epidemiológica. Fretta et al. (2017) destacam a importância da atividade física para reduzir os sinais e sintomas do climatério. $\mathrm{Na}$ presente pesquisa não houve diferença na prática de atividade física pelo grupo estudado ao final da intervenção, apesar do aumento da sua prevalência. Nesse sentido, destaca-se que a proposta desta intervenção deu mais ênfase na orientação das práticas alimentares num contexto de estilo de vida saudável, o que pode ser aprimorado em futuros estudos com a inclusão mais efetiva da atividade física, com intuito de avaliar o seu impacto na redução dos sintomas de climatério. Fato que pode ser atribuído as intervenções de EAN que abordaram questões referentes à saúde global, que faz referência à importância desse hábito.

Como ponto forte desse estudo citamos que trata-se de uma proposta de intervenção de EAN que mostrou resultados positivos com relação ao estado nutricional e consumo alimentar de mulheres na faixa etária correspondente a menopausa, fato que vai ao encontro de uma 
das conclusões apontadas na metanálise realizada por Hoga et al. (2015) que descreve que estratégias de enfrentamento para garantir apoio às mulheres durante a menopausa devem ser desenvolvidas de forma criativa e dinâmica, através da identificação e consideração das questões complexas envolvidas nesse processo.

Este estudo possui como limitação a duração da intervenção, que se deu em dois meses, devido ao tempo disponível pelos pesquisadores, o que pode ter implicado nas modificações não significativas, principalmente quanto aos sintomas do climatério.

\section{Conclusão}

A intervenção nutricional teve impacto positivo no estado nutricional, reduzindo índices de obesidade e medida da CC, que implicam em menor adiposidade abdominal e menor risco cardiometabólico. Também houve um incremento no consumo de lácteos, ovos, feijão, grãos e sementes e redução no consumo de pão branco, mostrando que a intervenção nutricional alterou positivamente os hábitos alimentares. Entretanto, não foram observadas mudanças significativas nos sintomas do climatério após a intervenção, fato que pode ser atribuído ao curto período da proposta de intervenção.

$\mathrm{O}$ desenvolvimento de mais pesquisas que envolvam mulheres no climatério pode contribuir para ampliar a percepção e cuidados de saúde, que podem impactar positivamente na qualidade de vida e redução de fatores de risco associados a problemas de saúde.
Impact of a food education intervention on the nutritional profile and food consumption standard in women in the climacteric

\section{Abstract}

Intervention strategies, within the scope of food and nutrition education, can contribute to the improvement of the food consumption pattern and nutritional status of women in the climacteric, resulting in health benefits and quality of life. The objective of the study was to evaluate the impact of a 2-month nutritional intervention based on food and nutrition education strategies on nutritional status, cardiovascular risk, climacteric symptoms, food intake and lifestyle of adult women. It is a quasi-experimental study. Data were collected, pre and post intervention, related to nutritional status (weight, height, body mass index (BMI) and waist circumference (WC)), lifestyle, satisfaction and body weight pattern and frequency of food intake. Climacteric symptoms were assessed using the Menopause Rating Scale (MRS). Data were analyzed using the Statistical Package for Social Science Program (SPSS). A total of sixteen women, with a mean age of $54 \pm 5.24$ years, whose $62.5 \%$ reported weight gain in recent years, and $75 \%$ expressed body dissatisfaction. There was a significant reduction in WC ( $p$ $=0.006)$ and BMI classification $(p=0.046)$ from pre to post intervention. There was an increase in the frequency of dairy products ingestion, eggs, beans, grains and reduction of white bread. As for lifestyle and climacteric symptoms, no significant changes were observed. The 2-month nutritional intervention showed a positive impact on food consumption habits and nutritional status of women in the climacteric period.

Keywords: Climacteric; Food and nutrition education; Nutritional status. 


\section{Referências}

ABDULNOUR, J.; DOUCET, E.; BROCHU, M.; LAVOIE J.-M.; STRYCHAR, I.; RABASA-LHORET, R.; PRUD'HOMME, D. The effect of the menopausal transition on body composition and cardiometabolic risk factors: a Montreal-Ottawa New Emerging Team group study. Menopause, v. 19, n. 7, p. 760-767, July 2012. doi: 10.1097/gme.0b013e318240f6f3

BARAZZETTI, L.; PATTUSSI, M. P.; GARCEZ, A. DA S.; MENDES, K. G.; THEODORO, H.; PANIZ, V. M. V.; OLINTO, M. T. A. Psychiatric disorders and menopause symptoms in Brazilian women. Menopause, v. 23 , n. 4 , p. $433-40$, 2016. Doi:10.1097/ gme.0000000000000548

BERNAUD, F.S.R.; RODRIGUES, T.C. Fibra alimentar - Ingestão adequada e efeitos sobre a saúde do metabolismo. Arquivos Brasileiros de Endocrinologia \& Metabologia, v. 57, n.6, 2013.

BRASIL. Ministério da Saúde. Secretaria de Atenção à Saúde. Departamento de Atenção Básica. Guia alimentar para a população brasileira/Ministério da Saúde, Secretaria de Atenção à Saúde, Departamento de Atenção Básica. 2. ed. 1. reimpr. - Brasília: Ministério da Saúde, 2014. 156 p. Disponível em: <http://bvsms.saude.gov.br/bvs/publicacoes/ guia_alimentar_populacao_brasileira_2ed. pdf $>$. Acesso em: 15 abr. 2019.

FERNANDES, C. E.; BARACAT, E. C.; LIMA, G. R. Climatério: manual de orientação. São Paulo: Ponto, 2004.

FRETTA, T. B.; REIS, N. M.; MACHADO, Z.; GUIMARÃES, A. C. DE A. Melhora dos sintomas do climatério por meio da atividade física: uma revisão sistemática. Saúde em Revista, v.17, n. 46, p. 67-78, 2017. Doi: 10.15600/2238-1244/sr.v17n46p67-78

GALLON, C.W.; WENDER, M. C. O. Estado nutricional e qualidade de vida da mulher climatérica. Revista Brasileira de Ginecologia e Obstetrícia, Caxias do Sul, v.34, n. 4, p 175-83, 2012. Doi: 10.1590/S010072032012000400007
GOMES, A. C. M.; DIAS, C. P.; GUERRA, R. O.; SALVO, V. L. M. A. DE. Impacto de estratégias de educação nutricional sobre variáveis antropométricas e conhecimento alimentar. Revista Brasileira em Promoção da Saúde, v. 26, n. 4, p.462-469, 2013. Doi: 10.5020/18061230.2013.p462

GU, J. W.; YOUNG, E.; PATTERSON, S. G.; MAKEY, K. L.; WELLS, J.; HUANG, M.; TUCKER, K. B.; MIELE, L. Postmenopausal obesity promotes tumor angiogenesis and breast cancer progression in mice. Cancer Biology \& Therapy, v. 11, n. 10, p. 910-917, 2011. Doi: 10.4161/cbt.11.10.15473

HOFFMANN, M.; MENDES, K. G.; CANUTO, R.; GARCEZ, A. DA S.; THEODORO, H.; RODRIGUES, A. D.; OLINTO, M. T. A. Padrões alimentares de mulheres no climatério em atendimento ambulatorial no Sul do Brasil. Ciência \& Saúde Coletiva, Rio de Janeiro, v. 20, n. 5, p. 1565-1574, May 2015. Doi: 10.1590/1413-81232015205.07942014.

HOGA, L.; RODOLPHO, J.; GONÇALVES, B.; QUIRINO, B. Women's experience of menopause: a systematic review of qualitative evidence the authors. JBI Database of Systematic Reviews and Implementation Reports, v. 13, n. 8, p. 250 - 337, 2015. Doi: 10.11124/jbisrir-2015-1948

IRMÃO, J. de S.; COSTA, M. de R. Conhecimento e hábitos de consumo de kefir na comunidade acadêmica da UFMS. Instituto de Laticínios Cândido Tostes, v. 73, n.1, p. 2736. 2018. Doi: 10.14295/2238-6416.v73i1.670

KLING, J. M., KELLY, M., RULLO, J., KAPOOR, E., KUHLE, C. L., VEGUNTA, S.; MARA, K. C.; FAUBION, S. S. Association between menopausal symptoms and relationship distress. Maturitas, v. 130, p. 1-5. 2019. Doi: 10.1016/j.maturitas.2019.09.006

KONING, L.; MERCHANT, A.T.; POGUE, J.; ANAND, S.S. Waist circumference and waist-to-hip ratio as predictors of cardiovascular events: meta-regression analysis of prospective studies. European Heart Journal, v. 28, n. 7, p. 850-856, 2007. Doi: 10.1093/eurheartj/ehm026 
MARTINAZZOI, J.; ZEMOLIN, G. P.; SPINELLI, R. B.; ZANARDO, V. P. S.; CENI, G. C. Avaliação nutricional de mulheres no climatério atendidas em ambulatório de nutrição no norte do Rio Grande do Sul, Brasil. Ciência \& Saúde Coletiva, v. 18, n. 11, p. 3349-3356, 2013. Doi: 10.1590/S1413-81232013001100024.

MONTEIRO, R. C. A.; RIETHER, P. T. A.; BURINI, R. C. Efeito de um programa misto de intervenção nutricional e exercício físico sobre a composição corporal e os hábitos alimentares de mulheres obesas em climatério. Revista de Nutrição, Campinas, v. 17, n. 4, p. 479-489, 2004. Doi: 10.1590/S1415-52732004000400008

MRS - THE MENOPAUSE RATING SCALE. Devloped by the Berlin center for Epidemiology and Heahh Research. [s.d]. Disponivel em: <http://www.menopause-rating-scale. info/>. Acesso em: 15 abr. 2019.

NETO, J. A. F.; FIGUERÊDO, E. D.; BARBOSA, J. B.; BARBOSA, F. F.; COSTA, G. R. D.; NINA, V. J. S.; NINA, R. V. DE A. H. Síndrome metabólica e menopausa: estudo transversal em ambulatório de ginecologia. Arquivos Brasileiros de Cardiologia, v. 95, n. 3, p. 339-45, 2010. Doi: 10.1590/S0066$782 \times 2010005000094$.

NOSSE, T. M.; MOREIRA, S. L. N.; ANDRADE, K. C. Avaliação dietética de mulheres climatéricas atendidas em uma clínica-escola de nutrição no município de São Paulo. $R e$ vista de Atenção à Saúde - RAS, v. 7, n. 21, 2009. Doi: 10.13037/rbcs.vol7n21.297

OMS- ORGANIZAÇÃO MUNDIAL DA SAÚDE. Investigaciones sobre la menopausia em los años noventa (Serie de Informes Técnicos, Vol. 866). Genebra: World Health Organization, 1996. Disponível em: https://apps.who. int/iris/bitstream/handle/10665/41984/WHO_ TRS_866_spa.pdf?sequence=1\&isAllowed =y

PEDRO, A. O.; PINTO-NETO, A. M.; COSTA-PAIVA, L. H. S.; OSIS, M. J. D.; HARDYA, E. E. Síndrome do climatério: inquérito populacional domiciliar em Campinas, SP. Revista de Saúde Pública, v. 37, n. 6, p.735-742, 2003. Doi: 10.1590/S0034-89102003000600008.
SILVA, M. A.; FRANCESCHI, R. J.; PAGANOTTO, M.; PASSONI, C. R. DE M. S. Perfil nutricional e sintomatológico de mulheres no climatério e menopausa. Cadernos da Escola de Saúde, v.2, n.8, p. 96-113, 2012.

VIDAL, A.M.; DIAS, D. O.; MARTINS, E. S. M.; OLIVEIRA, R. S.; NASCIMENTO, R. M. S.; CORREIA, M. DAS G. DA S. A ingestão de alimentos funcionais e sua contribuição para a diminuição da incidência de doenças. Cadernos de Graduação - Ciências Biológicas e da Saúde, v. 1, n.15, p. 43-52, out, 2012.

WHO - WORLD HEALTH ORGANIZATION. Expert Committee on Physical Status: Use and interpretatation of Anthropomety Physical status: the use and interpretation of anthropometry: report of a WHO expert committee. Geneva: WHO, 1995. Disponivel em: <https://apps.who.int/iris/bitstream/handle/10665/37003/WHO_TRS_854.pdf?sequence $=1 \&$ isAllowed $=y>$. Acesso em: 05 abr. 2019

WHO - WORLD HEALTH ORGANIZATION. Nutrition. Obesity: preventing and managing the global epidemic. Geneva: WHO, 1998. Disponível em: <https://www.who.int/nutrition/publications/obesity/WHO_TRS_894/ en/>. Acesso em: 05 abr. 2019.

ZAMPIERI, M. F. M.; TAVARES, C. M. A.; HAMES, M. DE L. C.; FALCON, G. S.; SILVA, A. L. DE; GONÇALVES, L. T. O processo de viver e ser saudável das mulheres no climatério. Escola Anna Nery, v. 13, n. 2, p. 305-12, abr-jun. 2009. Doi: 10.1590/S141481452009000200010

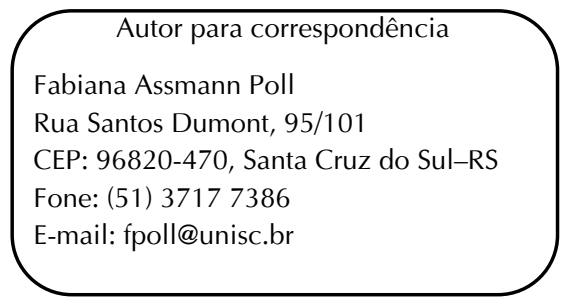

RBCEH, Passo Fundo, v. I7, n. I, p. 127- |42, jan./abr. 2020 